PROCEEDINGS OF THE

AMERICAN MATHEMATICAL SOCIETY

Volume 127, Number 3, March 1999, Pages 935-936

S 0002-9939(99)04946-1

\title{
DIMENSION OF A MINIMAL NILPOTENT ORBIT
}

\author{
WEIQIANG WANG
}

(Communicated by Roe Goodman)

\begin{abstract}
We show that the dimension of the minimal nilpotent coadjoint orbit for a complex simple Lie algebra is equal to twice the dual Coxeter number minus two.
\end{abstract}

Let $\mathfrak{g}$ be a finite dimensional complex simple Lie algebra. We fix a Cartan subalgebra $\mathfrak{h}$, a root system $\Delta \subset \mathfrak{h}^{*}$ and a set of positive roots $\Delta_{+} \subset \Delta$. Let $\rho$ be half the sum of all positive roots. Denote by $\theta$ the highest root and normalize the Killing form

$$
(,): \mathfrak{g} \times \mathfrak{g} \rightarrow \mathbb{C}
$$

by the condition $(\theta, \theta)=2$. The dual Coxeter number $h^{\vee}$ can be defined as $h^{\vee}=$ $(\rho, \theta)+1$ (cf. $[\mathrm{K}])$. This intrinsic number of the Lie algebra $\mathfrak{g}$ plays an important role in representation theory (cf. e.g. $[\mathrm{K}]$ ).

As is well known there exists a unique nonzero nilpotent (co)adjoint orbit of minimal dimension. A coadjoint orbit can be identified with an adjoint one by means of the Killing form. For more detail on the nilpotent orbits, we refer to the excellent exposition $[\mathrm{CM}]$ and the references therein. Our result of this short note is the following theorem.

Theorem 1. The dimension of the minimal nonzero nilpotent orbit equals $2 h^{\vee}-2$.

We start with the following well-known lemma; cf., for example, Lemma 4.3.5, $[\mathrm{CM}]$.

Lemma 1. The dimension of the minimal nonzero nilpotent orbit is equal to one plus the number of positive roots not orthogonal to $\theta$.

We call a root $\alpha$ in $\Delta_{+}$special if $\theta-\alpha$ is also a root. The subset of special roots, denoted by $\mathbb{S}$, was singled out in $[\mathrm{KW}, \mathrm{W}]$ for some other purposes. It is easy to see that we can also define the set $\mathbb{S}$ equivalently as follows.

Lemma 2. The set $\mathbb{S}$ is characterized by the property: $r_{\theta}(\alpha)=\alpha-\theta$, if $\alpha \in \mathbb{S}$; $r_{\theta}(\alpha)=\alpha$, if $\alpha \in \Delta_{+}-(\mathbb{S} \cup\{\theta\})$. In other words, $\mathbb{S} \cup\{\theta\}$ is the set of positive roots not orthogonal to $\theta$.

The following lemma is taken from $[\mathrm{KW}, \mathrm{W}]$. The simple proof given below follows $[\mathrm{W}]$.

Lemma 3. The number of special roots is $\# \mathbb{S}=2\left(h^{\vee}-2\right)$.

Received by the editors July 7, 1997.

1991 Mathematics Subject Classification. Primary 22E10; Secondary $17 B 20$.

The author was partially supported by NSF grant DMS-9304580.

(C)1999 American Mathematical Society 
Proof. Since $(\theta, \theta)=2$ and $(\rho, \theta)=h^{\vee}-1$, we have

$$
r_{\theta} \rho=\rho-\frac{2(\rho, \theta)}{(\theta, \theta)} \theta=\rho-\left(h^{\vee}-1\right) \theta .
$$

On the other hand, it follows from Lemma 2 that

$$
\begin{aligned}
r_{\theta} \rho & =r_{\theta}\left(\frac{1}{2} \sum_{\alpha \in \Delta_{+}} \alpha\right) \\
& =\frac{1}{2}\left(\sum_{\theta \neq \alpha \in \Delta_{+}} r_{\theta}(\alpha)-\theta\right) \\
& =\frac{1}{2}\left(\sum_{\theta \neq \alpha \in \Delta_{+}} \alpha-(\# \mathbb{S}) \theta-\theta\right) \\
& =\rho-\frac{1}{2}(\# \mathbb{S}+2) \theta .
\end{aligned}
$$

Thus this lemma follows by comparing the right hand sides of the equations (1) and (2).

By combining Lemmas 1, 2 and 3, we prove our theorem. We have an immediate corollary from Lemmas 2 and 3 .

Corollary 1. The length of the reflection $r_{\theta}$ is $l\left(r_{\theta}\right)=2 h^{\vee}-3$.

\section{REFERENCES}

[CM] D.H. Collingwood and W.M. McGovern, Nilpotent orbits in semisimple Lie algebras, Van Nostrand Reinhold Math. Series, 1992. MR 94j:17001

[K] V.G. Kac, Infinite-dimensional Lie algebras, Third edition, Cambridge University Press, 1990. MR 92k: 17038

[KW] V.G. Kac and W. Wang, Vertex operator superalgebras and their representations, Contemporary Mathematics, vol. 175, (1994) 161-191. MR 95k:17040

[W] W. Wang, Representations of vertex operator algebras and superalgebras, Massachusetts Institute of Technology Ph.D. thesis, 1995.

Max-Planck Institut für Mathematik, 53225 Bonn, Germany

Current address: Department of Mathematics, Yale University, New Haven, Connecticut 06520

E-mail address: wqwang@math.yale.edu 